\title{
Efficacy and Toxicity of Metronomic Capecitabine in Advanced Hepatocellular Carcinoma
}

\author{
Ashraf Farrag \\ Clinical Oncology Department, Assiut University Hospital, Assiut, Egypt. \\ Email: darshraffarrag@yahoo.com
}

Received November $21^{\text {st }}, 2011$; revised December $20^{\text {th }}, 2011$; accepted January $5^{\text {th }}, 2012$

\begin{abstract}
Background: Hepatocellular carcinoma (HCC) is a hypervascular tumor. Metronomic chemotherapy; the continuous administration of low-dose chemotherapy; has both cytotoxic and antiangiogenic effects with low toxicity profile. We evaluated the efficacy and toxicity of metronomic capecitabine (MC) in patients with advanced HCC. Patients and Methods: From May 2010, we enrolled pts with either metastatic or locally advanced diseases not candidate for ablative or locoregional treatment and have acceptable liver function. Patients received oral MC in dose of $1000 \mathrm{mg} / \mathrm{m}^{2}$ daily in a 21 days cycle without interruption till disease progression or toxicity. Results: The study cohort consisted of 22 patients with a median age of 63 years. The median number of cycles received was 3 cycles (range $1-9$ ). From 19 patients were evaluable for response we had 3 partial responders, 10 stable diseases and disease progression in 6 patients. Median time to progression (TTP) was 2.2 months (95\% CI $1.4-6.24)$ and median survival time (OS) was 4.8 months $(95 \%$ CI 1.8 - 7.9). For 20 patients evaluable for safety: no grade III/IV hematological toxic effects were observed. Non-hematological toxic effects included grade III vomiting and diarrhea in one patient and grade III hand-foot syndrome in one patient. There was no treatment-related mortality. Conclusions: Based on the observed response rate, TTP and OS; MC has a modest antitumor efficacy in pts with advanced HCC. However, due to its low toxicity profile it deserves further attention as a convenient, outpatient-based chemotherapy regimen.
\end{abstract}

Keywords: Metronomic Chemotherapy; Capecitabine; Hepatocellular Carcinoma

\section{Introduction}

Hepatocellular carcinoma (HCC) is the fifth most common cancer worldwide and the third most common cause of cancer-related death [1].

The epidemiology of HCC is characterized by marked demographic and geographic variations. In Egypt, HCC is not uncommon. In the year 2002-2003 cancer registry, liver malignancies accounts for $7.5 \%$ of all cancer cases. [2] Also in a single center evaluation in Egypt, HCC was reported to account for about $4.7 \%$ of chronic liver disease patients [3].

Unfortunately, only a few patients are eligible for radical treatments with curative intent (i.e., surgical resection, liver transplantation, percutaneous ablation). The majority of HCC patients $(>80 \%)$ presents with advanced or unresectable disease. Even for those with resected disease, the recurrence rate can be as high as $50 \%$ at 2 years [4]. Transhepatic Arterial Chemoembolization (TACE) is not feasible in some situation like portal vein thrombosis and illogical in case of extrahepatic spread. Therefore, a substantial proportion of patients with advanced HCC are

\footnotetext{
"The author declares that he has no conflict of interest.
}

in need for systemic therapy.

Sorafenib (a multikinase inhibitor which suppresses tumor cell proliferation and angiogenesis) is proven to demonstrate a large survival benefit in advanced HCC for selected patients with Child-Pugh class A, B liver function [5-7]. However, a subgroup analysis showed a relatively low survival benefit for sorafenib over placebo in patients with extrahepatic spread [5].

Although most of the chemotherapeutic agents failed to show encouraging results [4], some studies has demonstrated promising efficacy for combination chemotherapy in advanced HCC [8-10].

The pyrimidine antimetabolite 5-fluorouracil (5-FU) was the first reported chemotherapeutic agent tested in the treatment of HCC $[11,12]$. Capecitabine is a rationally designed, orally administered, tumor-selective fluoropyrimidine that mimics continuous infusion of 5-FU. Capecitabine is converted to 5-FU preferentially in tumor tissue by the enzyme thymidine phosphorylase $[13,14]$. Treatment with capecitabine showed $11 \%$ of overall response rate including one complete remission and $22 \%$ of disease control rate with tolerable toxicity in $37 \mathrm{HCC}$ patients with underlying liver cirrhosis [15]. A study in- 
dicated that mild-to-moderate liver dysfunction in patients with colorectal carcinoma liver metastases did not significantly affect capecitabine pharmacokinetics [16]. This finding suggests that capecitabine may be useful for patients with hepatobiliary carcinoma, including patients with mildly-to-moderately impaired hepatic function.

Metronomic chemotherapy is defined as continuous administration of low-dose cytotoxic drugs. It was found to be as effective as chemotherapeutic drugs given at the maximum tolerated dose (MTD) but with reduced toxicity $[17,18]$. Metronomic chemotherapy provides a way to inhibit tumor angiogenesis by targeting proliferating endothelial cells in tumor blood vessels and circulating endothelial cell precursors that are later integrated in tumor vasculature [19-21].

Metronomic chemotherapy can be considered as a variant of "dense-dose" treatment with the difference that its cumulative dose can be significantly lower than MTD-based chemotherapy [19]. It reduces the level of toxicity and decreases or abolishes the need of support treatments with growth factors to accelerate recovery of bone marrow. In spite of having lower cumulative dose of drug, metronomic chemotherapy exhibits a superior therapeutic effect in terms of increase survival than conventional schemes of MTD [19].

HCC is a hypervascular tumor. So, capecitabine if given in a metronomic schedule; its cytotoxic effect on $\mathrm{HCC}$ cells can be potentiated by an additional effect on tumor angiogenesis.

Also, in preliminary results of a study done by Brandi et al., metronomic capecitabine (MC) showed promising effect on advanced HCC patients with a good tolerability profile [22].

In this study we investigate the efficacy and toxicity of $\mathrm{MC}$ in patients with metastatic or locally advanced HCC not candidate for ablative or locoregional treatment modalities.

\section{Patients and Methods}

\subsection{Eligibility Criteria}

Patients with either histologically proven HCC or combination of liver cirrhosis, radiologically documented hypervascular liver tumor and alphafetoprotein (AFP) level $>200 \mathrm{ng} / \mathrm{ml}$ [23]. Patients had to have distant metastases (stage IV) or locally advanced disease not suitable for surgical resection, liver transplantation, local ablation or chemoembolization techniques.

Other eligibility criteria were bidimensionally measurable disease, age $>18$ years; World Health Organization (WHO) performance status (PS) 0 - 2; Child-Pugh score of A or B; adequate hepatic, renal and bone marrow function. Specifically, their bilirubin level had to be $2.0 \mathrm{mg} / \mathrm{dL}$ or lower, their aspartate transaminase level had to be five times or lower the institutional upper limit of normal, and their serum creatinine level had to be 2.0 $\mathrm{mg} / \mathrm{dL}$ or lower. Minimum hematologic parameters included an absolute neutrophil count (ANC) greater than or equal to $1.0 \times 10^{9} / \mathrm{L}$ and a platelet count of greater than or equal to $100 \times 10^{9} / \mathrm{L}$. Main exclusion criteria were Child-Pugh score of $\mathrm{C}$, severe cardiac and/or respiratory failure, concurrent malignancy. Patients provided written informed consent.

\subsection{Treatment}

The therapy consisted of MC orally in a dose of 1000 $\mathrm{mg} / \mathrm{m}^{2} /$ day without interruption. The cycle is repeated every 21 days.

Before study entry, all patients provided a complete medical history and underwent a physical examination, including an assessment of performance status, recent weight loss, and comorbidities. Laboratory studies included a complete blood count, differential count, platelet count, biochemical liver and renal function tests, AFP, chest radiograph, and an abdomen-pelvic computed tomographic scan.

During treatment, patients were evaluated for a brief history, physical examination, and toxicity assessment. A complete blood count, renal and liver functions were measured every 3 weeks, before each cycle. AFP levels were examined every 6 weeks. Measurable disease was assessed radiographically every 6 weeks.

Treatment was continued until one of the following criteria was met: disease progression, unacceptable toxicity, patient refusal. Patients stopped treatment for any reason were allowed to receive $2^{\text {nd }}$ line therapy on a case by case evaluation.

\subsection{Response and Toxicity Assessments}

All eligible patients who received two cycle of treatment (6 weeks) were considered evaluable for response. Complete response (CR) was defined as the disappearance of all radiological and clinical evidence of tumor for a minimum of 4 weeks. Partial response (PR) was defined as a $\geq 50 \%$ reduction in the sum of the products of the longest perpendicular diameters of all measurable lesions, with no new lesions appearing and none progressing for at least 4 consecutive weeks. If the reduction was between $25 \%$ and $49 \%$, the response was classified as a minor partial response. A patient was considered to have progressive disease (PD) if any new lesion appeared, if the tumor size increased by $\geq 25 \%$ over pretreatment measurements or if the patient experienced deterioration in the clinical status consistent with disease progression. A patient who failed to meet the definition of CR, PR or PD but remained on treatment for at least 2 cycles was classified as having stable disease (SD). 
All patients who received at least one cycle of treatment were considered evaluable for safety. Toxicity was assessed before every cycle in accordance with the National Cancer Institute-Common Toxicity Criteria (NCICTC) criteria.

\subsection{Statistical Analysis}

The primary end-point was the response rate and toxicity and the secondary measures were overall survival (OS), Progression free survival (PFS), Time to progression (TTP). TTP was calculated from the first cycle of chemotherapy. OS and PFS were calculated from the starting date of chemotherapy to the date of death, progression or the last follow-up. Survival rates and time to progression were assessed by the Kaplan-Meier method.

\section{Results}

\subsection{Patient Characteristics}

A total number of 22 consecutive patients with advanced HCC were accrued. The clinical characteristics of the enrolled patients are shown in Table 1. The median age of the patients was 63 years, ranging from 53 to 72 years. WHO performance status scores were 1 in 10 patients (45\%), 2 in 12 patients (55\%), all patients were males. Twelve patients (55\%) had Child-Pugh class A and 10 patients (45\%) had class B. Most patients (86.3\%) had abnormal serum AFP level $(>5 \mathrm{ng} / \mathrm{ml})$, with a median value of $877 \mathrm{ng} / \mathrm{ml}$ (range 1.0 - >100,000 ng/ml). Regarding tumor status 14 patients (64\%) had extrahepatic disease at presentation, $8(36 \%)$ bone metastases and 6 (27\%) nodal diseases. Seven patients (32\%) had main portal vein thromboses. Six $(27 \%)$ had minimal ascites. Before receiving our protocol, 6 patients had previous active treatment for HCC, 5 patients $(27 \%)$ received chemotherapy and one patient underwent TACE with disease progression after it.

All 22 patients received at least one cycle of MC. Three patients lost follow up early. Two of them didn't attend for $2^{\text {nd }}$ cycle so they were not assessed for either toxicity or response, and the $3^{\text {rd }}$ patient lost follow up after starting the $2^{\text {nd }}$ cycle and was not assessed for response. Finally, treatment toxicity was assessed in 20 patients, and 19 patients were evaluable for response evaluation.

\subsection{Tumor Response and Survival}

A total of 74 cycles of MC were given for our patients with a median of 3 cycles (range from 1 to 9 cycles). Antitumor response could be radiologically assessed in 19 of 22 patients. Three patients were lost to follow-up, and the treatment response could not be determined. The objective response rate was $16 \%$ with 3 partial response and no complete responses. Ten patients (53\%) had stable
Table 1. Patient characteristics.

\begin{tabular}{|c|c|}
\hline Characteristics & Values \\
\hline Total no. of patients & 22 \\
\hline Age: Median (Range) & $63(53-72)$ \\
\hline Sex: Male/females & $22 / 0$ \\
\hline Performance status $0 / 1 / 2$ & $0 / 11 / 11$ \\
\hline \multicolumn{2}{|l|}{ Diagnosis } \\
\hline - Biopsy or cytology & $14(64 \%)$ \\
\hline - $\quad \alpha$-Fetoprotein and Radiology & $8(36 \%)$ \\
\hline \multicolumn{2}{|l|}{$\alpha$-Fetoprotein } \\
\hline - $\quad<200 \mathrm{ng} / \mathrm{ml}$ & $7(32 \%)$ \\
\hline - $200-10,000$ & $9(41 \%)$ \\
\hline - $>10,000$ & $6(27 \%)$ \\
\hline \multicolumn{2}{|l|}{ Pretreatment laboratory data } \\
\hline - $\quad$ Bilirubin (mg/dl) & $1.1(0.3-1.79)$ \\
\hline - $\operatorname{Albumin}(\mathrm{g} / \mathrm{dl})$ & $3.1(2.1-3.7)$ \\
\hline - $\quad$ SGOT (U/l) & $57(26-163)$ \\
\hline - $\quad \operatorname{SGPT}(\mathrm{U} / \mathrm{l})$ & $25(12-103)$ \\
\hline - Platelet count $\left(\times 10^{3} / \mathrm{ml}\right)$ & $219(160-354)$ \\
\hline - Hemoglobin (g/dl) & $11.5(9.8-15.6)$ \\
\hline - Prothrombine concentration $\%$ & $86.5(55-100)$ \\
\hline Presence of ascites & $6(27 \%)$ \\
\hline Main portal vein thrombosis & $7(32 \%)$ \\
\hline Child classification $\mathrm{A} / \mathrm{B} / \mathrm{C}$ & $12 / 10 / 0$ \\
\hline TNM stage III/IV & $8 / 14$ \\
\hline \multicolumn{2}{|l|}{ Extrahepatic disease } \\
\hline - Lymph node & $6(27 \%)$ \\
\hline - Bone & $8(42 \%)$ \\
\hline \multicolumn{2}{|l|}{ Previous treatment } \\
\hline - Chemotherapy & $5(23 \%)$ \\
\hline - TACE & $1(4.5 \%)$ \\
\hline
\end{tabular}

SGOT $=$ serum glutamic oxaloacetic transaminase; $\mathrm{SGPT}=$ serum glutamic pyruvic transaminase; TACE $=$ transarterial chemoembolization.

disease; so disease control $(\mathrm{CR}+\mathrm{PR}+\mathrm{SD})$ was $69 \%$. The remaining 6 patients $(31 \%)$ had disease progression. One patient from the partial responders later experienced disease progression; and shifted to $2^{\text {nd }}$ line treatment. He received a total of 6 cycles with a TTP of 4.5 months. The median TTP for all evaluable patients was 2.2 months (95\% CI 1.4 - 6.24) and median survival time was 4.8 months $(95 \%$ CI $1.8-7.9)$. At 6 moths OS was $70 \%$ and PFS was $44 \%$ (Figure 1).

A univariate analysis was done to evaluate the effects of different prognostic factors on disease outcome in term of OS and PFS. The analysis examined the effect of age, performance status, and tumor stage, site of extra- hepatic disease, Child score, and presence of ascities, AFP, 


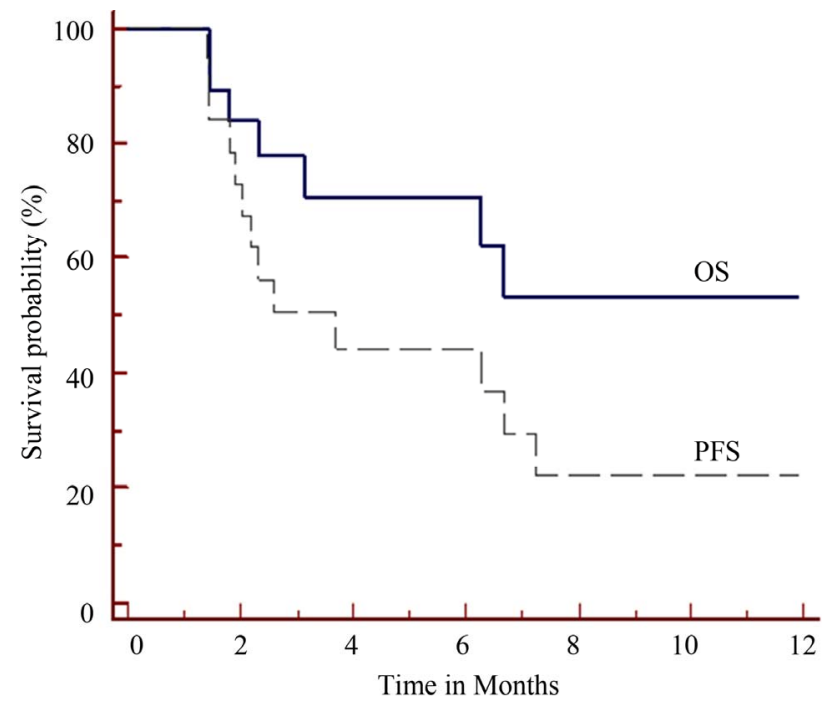

Figure 1. Survival results of the 19 patients evaluable for response. Solid line is for overall survival (OS) and dashed line is for progression free survival (PFS).

serum albumin, serum bilirubin, portal vein thrombosis, and hemoglobin and platelets counts. However none of the examined factors has a prognostic impact on the survival outcome.

\subsection{Toxicity}

No grade III/IV hematological toxic effects were observed; only grade I \& II thrombocytopenia $(20 \%)$ and grade I anemia (10\%). Non-hematological toxic effects included grade III vomiting and diarrhea in one patient (5\%) and grade III hand-foot syndrome in one patient $(5 \%)$ and grade I elevated hepatic enzymes $(20 \%)$. There was no treatment-related mortality.

\section{Discussion}

Advanced HCC is a challenging disease. Although the results of sorafenib are encouraging [5-7], it still has its limitations. The subgroup analysis demonstrated that patients with macroscopic vascular invasion and/or extrahepatic spread showed a smaller absolute survival benefit. However, these patients represent a major part of patients with advanced HCC. Cost and availability are additional limitation to the routine use of sorafenib especially in poor countries. Its high cost will prohibit treatment of many patients in need [24].

This warrants confirming the role of sorafenib for advanced $\mathrm{HCC}$ in various clinical settings. Additional studies of potential systemic chemotherapy regimens for the treatment of advanced HCC are also needed, especially after the promising results of some chemotherapeutic regimens [8-10].

In our study we evaluated oral capecitabine administrated in a metronomic schedule in patients with adv- anced HCC in a daily dose of $1000 \mathrm{mg} / \mathrm{m}^{2}$. The optimal metronomic dose is known for only few drugs and diseases but doses of MC closely similar to dose used in our study were found to be safe and effective in HCC and other malignancies. Brandi et al. studied $\mathrm{MC}$ in advanced HCC, Child A disease in a slightly different schedule where the first cycle was carried out with standard capecitabine ( $2000 \mathrm{mg} / \mathrm{m}^{2} /$ day; 14 over 21 days), followed by MC (1300 mg) without interruption. MC had a better tolerability than standard schedule and promises good efficacy [22]. A daily dose of $1300 \mathrm{mg} / \mathrm{m}^{2}$ of MC showed both good tolerability and efficacy in patients with advanced breast cancer [25]. A dose of capecitabine of 500 $\mathrm{mg}$ twice daily was shown to have activity and antiangiogenic effectiveness in colorectal cancer [26]. Also, a fixed daily dose of $1000 \mathrm{mg}$ of MC was found to be safe and valid treatment option in advanced colorectal and gastric cancer patients [27].

We had three PR and no CR with an objective response rate of $16 \%$ of the total 19 patients evaluable for response. Disease control was $69 \%$. These results are comparable to the result of $\mathrm{MC}$ in a phase II study done by Brandi et al where they had an objective response of $14.1 \%$ and disease control of $66.7 \%$ [28]. These results are slightly better than capecitabine when given in a conventional schedule of 2 weeks treatment every 21 days $\left(2000 \mathrm{mg} / \mathrm{m}^{2} / \mathrm{d}\right)$. It resulted in $11 \%$ overall response and $22 \%$ disease control. In another phase II study capecitabine combined with oxaliplatin in a 21 days cycle showed a $6 \%$ response rate and a $72 \%$ disease control rate in advanced HCC patients [29].

The response rate in our study is comparable to most of chemotherapy regimens. In advanced HCC the overall response rate to systemic chemotherapy is generally less than $10 \%$ [30]. This is probably owing to the strong multidrug-resistance gene expression usually observed in HCC cells [31]. Doxorubicin is the most widely investigated chemotherapeutic agent in advanced HCC. When given as a single agent, it has been shown to produce a response rate of about $10 \%-15 \%$, with no proven survival benefit [32]. When combined with cisplatin, 5-fluorouracil, and alpha-interferon (PIAF) it produced an overall response rate of $26 \%$, but considerable hematological toxicity and two treatment related deaths occurred [33].

Newer chemotherapeutic agents also produced comparable and sometimes lower response rates than our study. Several studies were done to evaluate the effects of taxanes [34,35], and gemcitabine [36,37] on advanced HCC. A combination of gemcitabine and oxaliplatin demonstrated an $18 \%$ response rate and $76 \%$ of patients had the disease under control [38]. However comparison between phase II studies is difficult due to different inclusion criteria, patients' characteristics and methods of response evaluation. 
In our study we had median TTP for all evaluable patients of 2.2 months (95\% CI $1.4-6.24$ ) and median survival time was 4.8 months (95\% CI 1.8 - 7.9). At 6 moths overall survival was $70 \%$ and progression free survival was $44 \%$. The low survival outcome observed here is similar to most chemotherapy agents who failed to show a survival benefit [4]. A higher survival outcome in some combination regimens (PIAF) was associated with a higher toxicity profile [33]. The survival outcome in our study is much less than the survival benefit obtained with the use of sorafenib [5-7]. The inclusion criteria in sorafenib trials were different, for example the SHARP trial was conducted on the Child-pugh class A patients who have a preserved liver function [5].

Categorizing our patients into different groups to evaluate the effect of different prognostic factors on outcome was done. However none of the examined variables demonstrated a significant difference on outcome. This may be due to the limited number of our patients.

The toxicity profile of MC was very low with only two cases of grade III non hematologic toxicity. This makes $\mathrm{MC}$ very tolerable especially in patients with impaired liver function.

The low toxicity profile and the convenient oral outpatient administration of MC may suggest its use in combination with other chemotherapeutic agents or a new targeted antiangiogenic. The results of a recent preclinical study highlight the possibility that even if metronomic chemotherapy per se fails to cause a clinical benefit, combination with a targeted antiangiogenic drug could yield results superior to the antiangiogenic drug used alone [39]. MC may be beneficial as a second line treatment. A recent study found that $\mathrm{MC}$ is well tolerated and can be active in HCC patients after progression under sorafenib and the extent of clinical benefit is going to be determined in the final analysis [40].

\section{Conclusion}

Based on the observed response rate, TTP and survival results; $\mathrm{MC}$ has a modest antitumor efficacy in pts with advanced HCC. These results are similar to most chemotherapeutic agents. However, due to its low toxicity profile it deserves further attention as a convenient, outpatient-based chemotherapy regimen. These advantages suggest for the future evaluation of $\mathrm{MC}$ in combination with other chemotherapeutic agents or with antiangiogenic target therapy known to have activity in HCC. A second line treatment after antiangiogenic therapy could be another area of interest for the study of MC.

\section{Acknowledgements}

The author is grateful to Dr Ahmed Marzouk, Dr Mohamed Wahman and Dr Ihsan Matar for their help and support during the preparation of this study.

\section{REFERENCES}

[1] D. M. Parkin, F. Bray, J. Ferlay, et al., "Estimating the World Cancer Burden: Globocan 2000," International Journal of Cancer, Vol. 94, No. 2, 2001, pp. 153-156. doi:10.1002/ijc. 1440

[2] National Cancer Institute, "Egypt Cancer Registry 20022003," Cairo University, Accessed on 2 August 2011. http://www.nci.cu.edu.eg/lectures/NCI\%20registry\%2020 02-03.pdf

[3] A. El-Zayadi, H. Abaza, S. Shawky, et al., "Prevalence and Epidemiological Features of Hepatocellular Carcinoma in Egypt-A Single Center Experience," Hepatology Research, Vol. 19, No. 2, 2001, pp. 170-179. doi:10.1016/S1386-6346(00)00105-4

[4] A. X. Zhu, "Systemic Therapy of Advanced Hepatocellular Carcinoma: How Hopeful Should We Be?" Oncologist, Vol. 11, No. 7, 2006, pp. 790-800.

doi:10.1634/theoncologist.11-7-790

[5] J. M. Llovet, S. Ricci, V. Mazzaferro, et al., "Sorafenib in Advanced Hepatocellular Carcinoma," The New England Journal of Medicine, Vol. 359, No. 4, 2008, pp. 378-390. doi:10.1056/NEJMoa0708857

[6] A. Cheng, Y. K. Kang, Z. Chen, et al., "Efficacy and Safety of Sorafenib in Patients in Asia-Pacific Region with Advanced Hepatocellular Carcinoma: A Phase III Randomised, Double-Blind, Placebo-Controlled Trial," The Lancet Oncology, Vol. 10, No. 1, 2009, pp. 25-34. doi:10.1016/S1470-2045(08)70285-7

[7] G. K. Abou-Alfa, L. Schwartz, et al., "Phase II Study of Sorafenib in Patients with Advanced Hepatocellular Carcinoma," Journal of Clinical Oncology, Vol. 24, No. 26, 2006, pp. 4293-4300. doi:10.1200/JCO.2005.01.3441

[8] M. Nakamura, H. Nagano, S. Marubashi, et al., "Pilot Study of Combination Chemotherapy of S-1, a Novel Oral DPD Inhibitor, and Interferon- $\alpha$ for Advanced Hepatocellular Carcinoma with Extrahepatic Metastasis," Cancer, Vol. 112, No. 8, 2008, pp. 1765-1771. doi:10.1002/cncr.23356

[9] T. Urabe, S. Kaneko, E. Matsushita, et al., "Clinical Pilot Study of Intrahepatic Arterial Chemotherapy with Methotrexate, 5-Fluorouracil, Cisplatin and Subcutaneous Interferon-Alpha-2b for Patients with Locally Advanced Hepatocellular Carcinoma," Oncology, Vol. 55, No. 1, 1998, pp. 39-47. doi:10.1159/000011833

[10] S. Obi, H. Yoshida, R. Toune, et al., "Combination Therapy of Intraarterial 5-Fluorouracil and Systemic Interferon-Alpha for Advanced Hepatocellular Carcinoma with Portal Venous Invasion," Cancer, Vol. 106, No. 9, 2006, pp. 1990-1997. doi:10.1002/cncr.21832

[11] M. A. Friedman, "Primary Hepatocellular Cancer-Present Results and Future Prospects," International Journal of Radiation Oncology Biology Physics, Vol. 9, No. 12, 1983, pp. 1841-1850.

[12] D. Y. Lin, S. M. Lin and Y. F. Liaw, "Non-Surgical Treatment of Hepatocellular Carcinoma," Journal of Gastro- 
enterology and Hepatology, Vol. 12, No. 9-10, 1997, pp. S319-S328. doi:10.1111/j.1440-1746.1997.tb00516.x

[13] M. Miwa, M. Ura, M. Nishida, et al., "Design of a Novel Oral Fluoropyrimidine Carbamate, Capecitabine, Which Generates 5-Fluorouracil Selectively in Tumors by Enzymes Concentrated in Human Liver and Cancer Tissue," European Journal of Cancer, Vol. 34, No. 8, 1998, pp. 1274-1281. doi:10.1016/S0959-8049(98)00058-6

[14] J. Schuller, J. Cassidy, E. Dumont, et al., "Preferential Activation of Capecitabine in Tumor Following Oral Administration to Colorectal Cancer Patients," Cancer Chemotherapy and Pharmacology, Vol. 45, No. 4, 2000, pp. 291-297. doi:10.1007/s002800050043

[15] Y. Z. Patt, M. M. Hassan, A. Aguayo, et al., "Oral Capecitabine for the Treatment of Hepatocellular Carcinoma, Cholangiocarcinoma, and Gallbladder Carcinoma," Cancer, Vol. 101, No. 3, 2004, pp. 578-586. doi:10.1002/cncr.20368

[16] C. Twelves, R. Gynne-Jones, J. Cassidy, et al., "Effect of Hepatic Dysfunction Due to Liver Metastases on the Pharmacokinetics of Capecitabine and Its Metabolites," Clinical Cancer Research, Vol. 5, 1999, pp. 1696-1702.

[17] J. Folkman, "New Perspectives in Clinical Oncology from Angiogenesis Research," European Journal of Cancer, Vol. 32, No. 14, 1996, pp. 2534-2539. doi:10.1016/S0959-8049(96)00423-6

[18] U. Emmenegger, S. Man, Y. Shaked, et al., "A Comparative Analysis of Low-Dose Metronomic Cyclophosphamide Reveals Absent or Low-Grade Toxicity on Tissues Highly Sensitive to the Toxic Effects of Maximum Tolerated Dose Regimens," Cancer Research, Vol. 64, No. 11, 2004, pp. 3994-4000. doi:10.1158/0008-5472.CAN-04-0580

[19] R. S. Kerbel and B. A. Kamen, "The Antiangiogenicbasis of Metronomic Chemotherapy," Nature Reviews Cancer, Vol. 4, No. 6, 2004, pp. 423-436. doi:10.1038/nrc1369

[20] Y. Shaked, F. Bertolini, S. Man, et al., "Genetic Heterogeneity of the Vasculogenic Phenotype Parallels Angiogenesis; Implications for Cellular Surrogate Marker Analysis of Antiangiogenesis," Cancer Cell, Vol. 7, No. 1, 2005, pp. 101-111. doi:10.1016/S1535-6108(04)00369-1

[21] F. Bertolini, S. Paul, P. Mancuso, et al., "Maximum Tolerable Dose and Low-Dose Metronomic Chemotherapy Have Opposite Effects on the Mobilization and Viability of Circulating Endothelial Progenitor Cells," Cancer Research, Vol. 63, No. 15, 2003, pp. 4342-4346.

[22] G. Brandi, S. Fanello, F. Piscaglia, et al., "Metronomic Capecitabine in Advanced Patients with Hepatocellular Carcinoma (HCC): Preliminary Results," Journal of Clinical Oncology, Vol. 25, No. 18S, 2007, p. 15163.

[23] J. Bruix and M. Sherman, "Management of Hepatocellular Carcinoma," Hepatology, Vol. 42, No. 5, 2005, pp. 1208-1236. doi:10.1002/hep.20933

[24] P. R. Galle, "Sorafenib in Advanced Hepatocellular Carcinoma-We Have Won a Battle Not the War," Journal of Hepatology, Vol. 49, No. 5, 2008, pp. 871-873. doi:10.1016/j.jhep.2008.09.001

[25] M. R. Stockler, T. Sourjina, P. V. Grimison, et al., "A Ran- domized Trial of Capecitabine (C) Given Intermittently (IC) Rather Than Continuously (CC) Compared to Classical CMF as First-Line Chemotherapy for Advanced Breast Cancer (ABC)," Journal of Clinical Oncology, Vol. 25, No. 18S, 2007, p. 1031.

[26] S. Steinbild, J. Arends, M. Medinger, et al., "Metronomic Antiangiogenic Therapy with Capecitabine and Celecoxib in Advanced Tumor Patient-Results of a Phase II Study," Onkologie, Vol. 30, No. 12, 2007, pp. 629-635.

[27] M. Nannini, E. Nobili, R. Di Cicilia, et al., "Widen the Setting of Cancer Patients Who Could Benefit from Metronomic Capecitabine," Cancer Chemotherapy and Pharmacology, Vol. 64, No. 1, 2009, pp. 189-193. doi:10.1007/s00280-009-0930-Z

[28] G. Brandi, V. Agostini, F. de Rosa, et al., "Metronomic Capecitabine in Child-Pugh a Patients with Unresectable Hepatocellular Carcinoma," Gastrointestinal Cancer Symposium, Orlando, 22-24 January 2010.

[29] V. Boige, J. L. Raoul, J. P. Pignon, et al., "Multicentre Phase II Trial of Capecitabine plus Oxaliplatin (XELOX) in Patients with Advanced Hepatocellular Carcinoma: FFCD 03-03 Trial," British Journal of Cancer, Vol. 97, 2007, pp. 862-867

[30] A. K. Nowak, P. K. Chow and M. Findlay, "Systemic Therapy for Advanced Hepatocellular Carcinoma: A Review," European Journal of Cancer, Vol. 40, No. 10, 2004, pp. 1474-1484. doi:10.1016/j.ejca.2004.02.027

[31] I. O. Ng, C. L. Liu, S. T. Fan, et al., "Expression of P-Glycoprotein in Hepatocellular Carcinoma. A Determinant of Chemotherapy Response," American Journal of Clinical Pathology, Vol. 113, No. 3, 2000, pp. 355-363.

[32] E. C. Lai, T. K. Choi, C. H. Cheng, et al., "Doxorubicin for Unresectable Hepatocellular Carcinoma. A Prospective Study on the Addition of Verapamil," Cancer, Vol. 66, No. 8, 1990, pp. 1685-1687. doi:10.1002/1097-0142(19901015)66:8<1685::AID-CNC R2820660805>3.0.CO;2-W

[33] T. W. Leung, Y. Z. Patt, W. Y. Lau, et al., "Complete Pathological Remission Is Possible with Systemic Combination Chemotherapy for Inoperable Hepatocellular Carcinoma," Clinical Cancer Research, Vol. 5, No. 7, 1999, pp. 1676-81.

[34] Y. Chao, W. K. Chan, M. J. Birkhofer, et al., "Phase II and Pharmacokinetic Study of Paclitaxel Therapy for Unresectable Hepatocellular Carcinoma Patients," British Journal of Cancer, Vol. 78, No. , 1998, pp. 34-39. doi:10.1038/bjc. 1998.438

[35] M. Hebbar, O. Ernst, S. Cattan, et al., "Phase II Trial of Docetaxel Therapy in Patients with Advanced Hepatocellular Carcinoma," Oncology, Vol. 70, No. 2, 2006, pp. 154-158. doi:10.1159/000093007

[36] T. S. Yang, C. H. Wang, R. K. Hsieh, et al., "Gemcitabine and Doxorubicin for the Treatment of Patients with Advanced Hepatocellular Carcinoma: A phase I-II Trial," Annals of Oncology, Vol. 13, No. 11, 2002, pp. 1771-1778. doi:10.1093/annonc/mdf303

[37] Z. Guan, Y. Wang, S. Maoleekoonpairoj, et al., "Prospective Randomised Phase II Study of Gemcitabine at Standard or Fixed Dose Rate Schedule in Unresectable Hepa- 
tocellular Carcinoma," British Journal of Cancer, Vol. 89, No. 10, 2003, pp. 1865-1869. doi:10.1038/sj.bjc.6601369

[38] S. Louafi, V. Boige, M. Ducreux, et al., "Gemcitabine plus Oxaliplatin (GEMOX) in Patients with Advanced Hepatocellular Carcinoma (HCC): Results of a Phase II Study," Cancer, Vol. 109, No. 7, 2007, pp. 1384-1390. doi:10.1002/cncr.22532

[39] T. C. Tang, S. Man, C. R. Lee, et al., "Impact of Metronomic UFT/Cyclophosphamide Chemotherapy and Anti- angiogenic Drug Assessed in a New Preclinical Model of Locally Advanced Orthotopic Hepatocellular Carcinoma," Neoplasia, Vol. 12, No. 3, 2010, pp. 264-274.

[40] F. de Rosa, V. Agostini, S. Di Girolamo, et al., "Metronomic Capecitabine as Second-Line Treatment for Patients with Hepatocellular Carcinoma with Preserved Liver Function: A Phase II Study," ASCO Annual Meeting, Chicago, 1-5 June 9, 2011, p. e14608. 